\title{
Entre rutinas cotidianas y eventos extraordinarios: las construcciones de conceptos temporales en la sociedad maya prehispánica
}

Between daily routines and extraordinary events: constructions of temporal concepts in pre-Hispanic Mayan society

Entre routines quotidiennes et événements extraordinaires : la construction de concepts temporels au sein de la société maya préhispanique

Takeshi Inomata

\section{OpenEdition}

Journals

Edición electrónica

URL: http://journals.openedition.org/jsa/15417

DOI: $10.4000 /$ jsa. 15417

ISSN: 1957-7842

Editor

Société des américanistes

Referencia electrónica

Takeshi Inomata, «Entre rutinas cotidianas y eventos extraordinarios: las construcciones de

conceptos temporales en la sociedad maya prehispánica », Journal de la société des américanistes [En línea], Maya times | 2017, Publicado el 31 diciembre 2017, consultado el 14 noviembre 2019. URL http://journals.openedition.org/jsa/15417 ; DOI : 10.4000/jsa.15417 


\title{
Entre rutinas cotidianas y eventos extraordinarios: las construcciones de conceptos temporales en la sociedad maya prehispánica
}

\author{
Takeshi InOMATA*
}

El simbolismo de los Grupos de tipo E presentes en el sur de Mesoamérica se transformó durante el período Preclásico. Durante el Preclásico Temprano y la primera parte del Preclásico Medio, los complejos arquitectónicos y los escondites de hachas de piedra verde, en la Costa Sur del Golfo y en Chiapas, se referían a símbolos terrenales. Pero, con el desarrollo de la agricultura y del cultivo del maíz durante el Preclásico Medio, los Grupos de tipo E y los depósitos rituales en el área maya se fueron vinculando más fuertemente con los movimientos del sol y el simbolismo de las direcciones cardinales. Los datos recabados indican que prácticas sociales modelaron las concepciones simbólicas y temporales, que iban asociadas con los complejos arquitectónicos monumentales. Los rituales públicos y los proyectos colectivos de construcción ofrecieron probablemente oportunidades para que una misma temporalidad fuese compartida por diversos miembros de comunidades, quienes, en otros contextos, seguían diferentes ritmos de vida. [Palabras claves: Maya, Preclásico, concepciones del tiempo, rituales, Grupos de tipo E, simbolismo de las direcciones.]

Between daily routines and extraordinary events: constructions of temporal concepts in pre-Hispanic Mayan society. The symbolisms of E-Group assemblages in southern Mesoamerica transformed during the Preclassic period. Architectural complexes and greenstone axe caches on the southern Gulf Coast and in Chiapas during the Early Preclassic and the early part of the Middle Preclassic emphasized references to terrestrial symbols. With the development of maize agriculture during the Middle Preclassic, E-Group assemblages in the Maya area and related ritual deposits came to be tied more closely to the movements of the sun and the symbolism of cardinal directions. These data indicate that social practices shaped the construction of symbolic and temporal concepts associated with the architectural complexes. Public rituals and communal construction projects probably offered opportunities to share the same temporality among diverse members of the community, who followed different rhythms of life in other contexts. [Key words: Maya, Preclassic, concepts of time, rituals, E-Group, directional symbolism.]

* Universidad de Arizona, Tucson, USA [inomata@email.arizona.edu]. 
Entre routines quotidiennes et événements extraordinaires : la construction de concepts temporels au sein de la société maya préhispanique. En Mésoamérique méridionale, le symbolisme des groupes dits « de type E», s'est transformé durant la période préclassique. Au sud de la côte du Golfe et au Chiapas, les complexes architecturaux et les caches composées de haches de pierre verte datant du Préclassique ancien et des premières phases du Préclassique moyen étaient liés à des symboles terrestres. Avec le développement de la culture du maïs au cours du Préclassique moyen, les Groupes E de l'aire maya et les dépôts rituels associés ont été plus nettement reliés aux mouvements du soleil et au symbolisme des quatre directions. Les données recueillies indiquent que les pratiques sociales ont modelé les conceptions symboliques et temporelles associées aux complexes architecturaux monumentaux. Les rituels publics et les édifications de monuments offraient aux membres de communautés dont les rythmes de vie divergeaient par ailleurs la possibilité de partager des temporalités communes. [Mots-clés : Maya, Préclassique, conceptions du temps, rituels, Groupes de type E, symbolisme des directions.]

Se considera comúnmente la concepción del tiempo entre los mayas como cíclica y se contrasta con otra concepción, lineal, la cual sería propia de la sociedad occidental. Sin embargo, las concepciones temporales de las diferentes sociedades generalmente combinan elementos cíclicos y lineales, y una categorización simple y unívoca resulta problemática. Por ejemplo, muchas sociedades, incluyendo el mundo occidental, utilizan calendarios lunisolares, los cuales combinan la naturaleza cíclica de los movimientos de la luna y el sol: estos calendarios, junto con los estrictamente solares, están estrechamente vinculados con los ciclos de las estaciones y de las actividades agrícolas. Por su lado, los calendarios mayas presentan características interesantes. El calendario de 260 días tiene un aspecto fuertemente cíclico, pero su duración no corresponde al ciclo del año. La Cuenta Larga, por su parte, conlleva un concepto al parecer más lineal, pero a la vez incorpora un aspecto cíclico. Ahora bien, su base (cíclica) de 360 días genera discrepancias notables con el ciclo del año, observables incluso a escala de la vida de una sola y misma persona. Entonces, en lugar de caracterizar simplemente como cíclica o linear la concepción del tiempo de una sociedad dada, es más adecuado analizar cómo pudieron integrarse diferentes elementos temporales (incluyendo lo cíclico y lo linear), y cómo se construyeron las percepciones temporales en relación con las actividades sociales.

En su crítica de la categorización simplista de los conceptos temporales por parte de las sociedades, Maurice Bloch (1977) argumentó que estos conceptos en Bali se dividen en dos tipos: el tiempo ritual, que es cíclico y que va vinculado con actividades rituales, las cuales a su vez están ligadas a la legitimización de la autoridad política; y el tiempo mundano, lineal, que está basado en rutinas diarias, y tiene un carácter universal ya que está relacionado con la experiencia de los cambios de estaciones y otros fenómenos naturales. Richard Bradley (1991), 
por su parte, adoptó los conceptos de tiempo ritual y de tiempo mundano para dar cuenta del período neolítico de Gran Bretaña. Sin embargo, esta visión de la combinación de diferentes conceptos temporales dentro de una sociedad también ha suscitado críticas. Así, varios investigadores apuntaron que actos rituales y mundanos pueden incorporar simultáneamente elementos repetitivos y secuenciales y, de esa manera, ciclicidad y linealidad se integran en un solo concepto (Gell 1992; Howe 1981; Munn 1992). A su vez, la experiencia de fenómenos naturales no desemboca en una concepción universal del tiempo. Estas discusiones indican la importancia de examinar cómo diferentes elementos temporales se van integrando, cómo estos conceptos condicionan actividades sociales y cómo, a la inversa, los actos de la gente construyen concepciones temporales.

Basado en estas consideraciones, el presente artículo revisa el desarrollo de conceptos temporales en el mundo maya antiguo, utilizando principalmente unos datos arqueológicos del período preclásico encontrados en el sitio maya de Ceibal y en otras ruinas relacionadas.

\section{Ceibal}

El sitio de Ceibal está ubicado al suroeste del departamento del Peten en Guatemala, en la orilla del río Pasión (Figura 1, página siguiente). Ceibal ha ocupado un lugar especial en la arqueología maya desde las investigaciones pioneras de la Universidad de Harvard en los años 1960 (Graham 1990; Sabloff 1975; Smith 1982; Tourtellot 1988; Willey 1990). La excavación intensiva de sus complejos ceremoniales, los Grupos A, C y D, proporcionó datos significativos sobre su ocupación larga, desde los principios del Preclásico Medio hasta los finales del Clásico Terminal (1000 a.C.-900 d.C.). Por su parte, el recorrido extensivo de las áreas periféricas reveló el proceso de expansión del asentamiento, mientras que el análisis detallado de la cerámica estableció una cronología que ha servido de base para las investigaciones subsecuentes en las regiones cercanas. Partiendo de los resultados de aquellas investigaciones e incorporando los avances de la arqueología maya y de la epigrafía, iniciamos el proyecto arqueológico Ceibal-Petexbatun en el año 2005, enfocándonos en algunas preguntas específicas sobre los procesos de cambios sociales y políticos. Uno de los focos de ese estudio era la transformación de la comunidad de Ceibal durante el período preclásico (Inomata et al. 2010, 2013; Inomata 2014a, 2014b).

Las excavaciones de Harvard se habían centrado sobre el período clásico, incluyendo los elementos más tempranos de las construcciones de aquella época. En sus trabajos, los arqueólogos habían detectado varios pisos debajo de las plazas del Grupo A, y establecido que dicho conjunto ceremonial había sido el foco principal de la ocupación más temprana de Ceibal. Sin embargo, 
no se había descubierto más que tres muros, y no se había localizado ninguna estructura substancial del Preclásico Medio (Smith 1982, p. 224, 227).

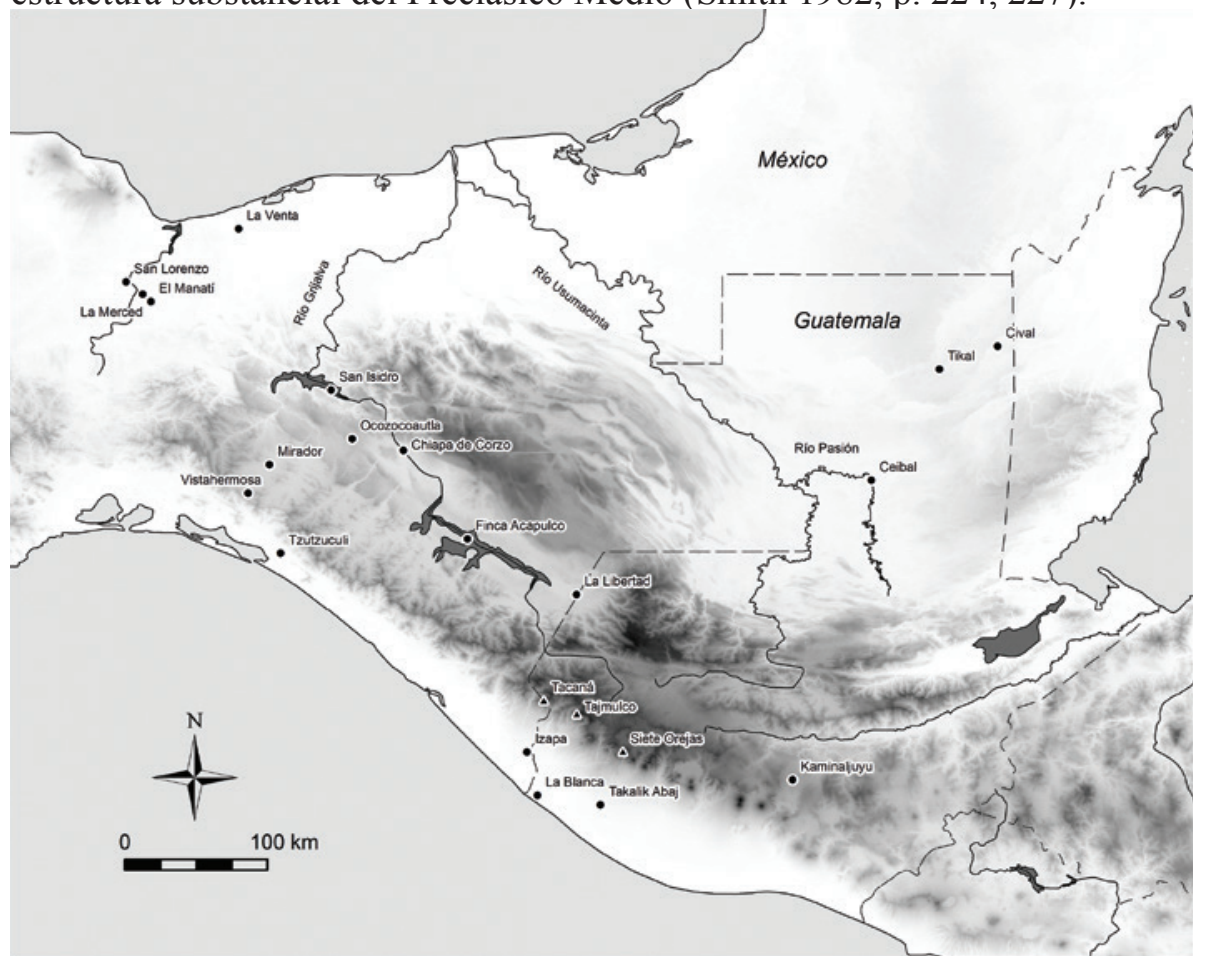

Fig. 1 - Mapa del área maya y la región del Istmo con las ubicaciones de los sitios y volcanes mencionados en el texto.

Por nuestra parte realizamos excavaciones profundas principalmente en el Grupo A para examinar los depósitos del período preclásico. Aparecieron numerosos episodios de construcciones de edificios, los cuales empiezan al principio del Preclásico Medio. Se ha podido refinar la cronología del sitio a partir del análisis de la cerámica y más de cien dataciones de radiocarbono (Inomata et al. 2013, 2015a, 2015b). La Figura 2 señala las fases establecidas para Ceibal en relación con las secuencias cronológicas de otros sitios o regiones. Las épocas que se discuten en este texto abarcan el Preclásico Medio, el Preclásico Tardío y el Preclásico Terminal o Protoclásico.

\section{EI desarrollo del Grupo de tipo E durante el Preclásico Medio}

Las excavaciones en la Plaza Central del Grupo A de Ceibal pusieron a la luz un complejo ceremonial formal que es comúnmente llamado "Grupo de tipo E" 
Entre rutinas cotidianas y eventos extraordinarios

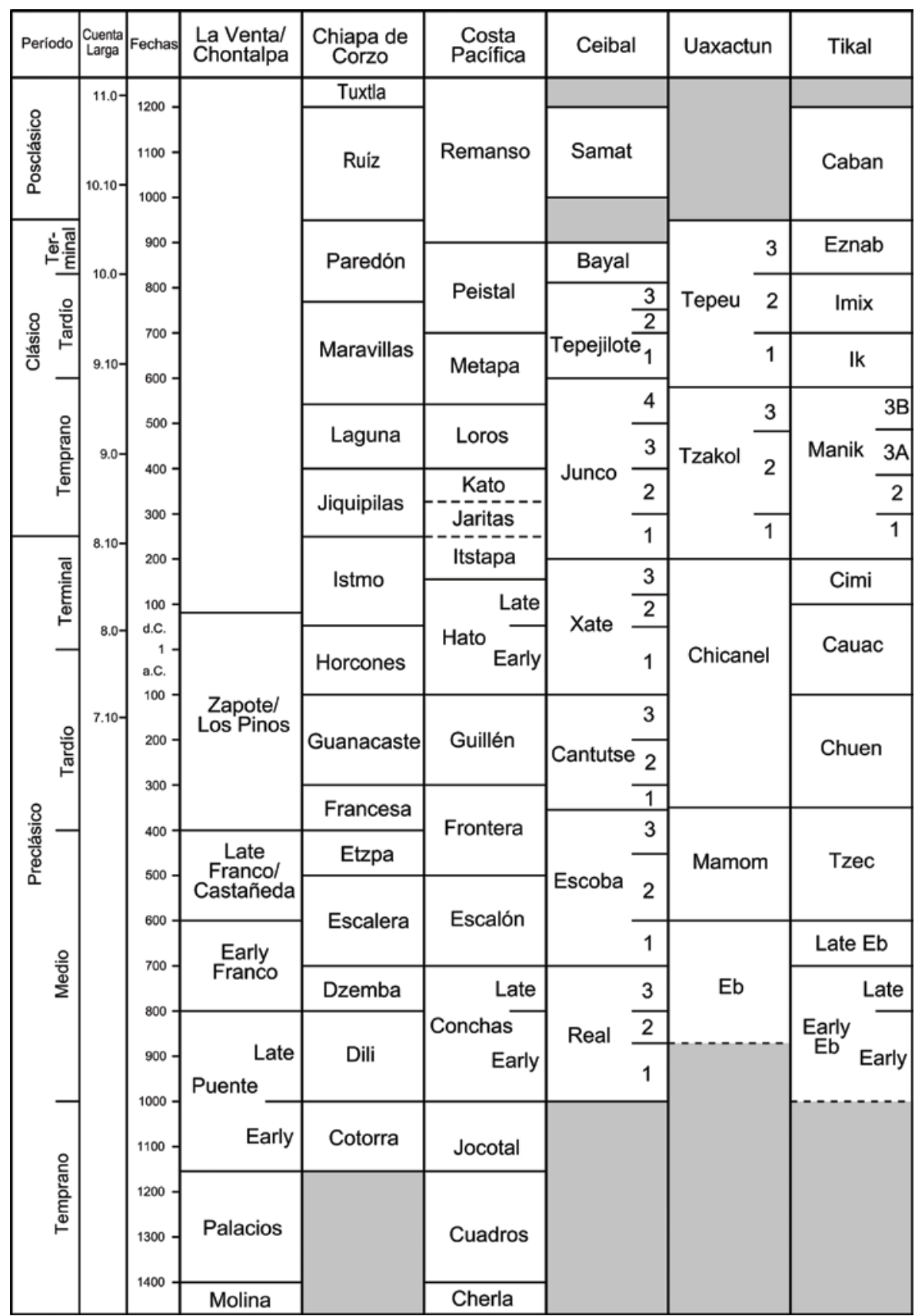

Fig. 2 - Cronología de Ceibal y otras regiones relacionadas. 
y consiste en una estructura cuadrada al oeste y una plataforma alargada al este (Figura 3). Se fundó este grupo en los inicios del asentamiento durante la fase Real 1 alrededor de 950 a.C., lo que hace de esta construcción el Grupo de tipo E más temprano conocido en toda Mesoamérica. Para la edificación de este conjunto, los residentes de Ceibal rasparon el suelo natural del área, utilizando la capa original de sascab como primer piso de la plaza. Se formó la plataforma este, que llamamos "Xa'an", tallando la capa natural de sascab. Para el edificio oeste, al cual se dio el nombre de "Ajaw", se agregó un suelo negro encima de un afloramiento elevado de sascab que fue tallado.

El eje central este-oeste del Grupo de tipo E de Ceibal fue el foco de actividades rituales a lo largo del período preclásico. Ahí se encontraron numerosos escondites de hachas de piedra verde y otros tipos de ofrendas. Al suroeste del Grupo de tipo E existió una plataforma grande, Sulul, la cual probablemente fue construida al mismo tiempo que el Grupo de tipo E, alrededor de 950 a.C. No se hallaron estructuras residenciales en las áreas expuestas de Sulul, y, por tanto, no queda claro si esta plataforma sirvió o no como base para un conjunto residencial en la parte temprana del Preclásico Medio. Alrededor de 800 a.C., a principios de la fase Real 3, otra plataforma grande, K'at, fue agregada al noreste del Grupo de tipo E. La presencia de múltiples edificios encima de esta plataforma sugiere que ella, sí, funcionó como un complejo residencial de la élite emergente. Durante la parte tardía del Preclásico Medio, o fase Escoba (700-350 a.C), varias estructuras fueron construidas encima de la Plataforma Ch'och', la cual representa una versión más tardía de Sulul.

Esta configuración que combina un Grupo de tipo E con plataformas grandes colocadas al norte y al sur, así como el hallazgo de escondites de hachas de piedra verde, recuerdan mucho a diversos asentamientos contemporáneos de Chiapas, incluyendo Chiapa de Corzo (Bachand y Lowe 2012; Hicks y Rosaire 1960; Lowe 1962; Lowe y Agrinier 1960; Mason 1960), Ocozocoautla (McDonald 1999, p. 61-62), San Isidro (Lowe 1981), La Libertad (Miller 2014), Mirador (Agrinier 1975, 2000), Vistahermosa (Treat 1986) y Tzutzuculi (McDonald 1983) y el centro olmeca de La Venta (Clark y Hansen 2001; Lowe 1977). John Clark (Clark y Hansen 2001) llama esta configuración estandarizada como patrón de "Chiapas Formativo Medio" (CFM).

Muchos arqueólogos sugieren que los Grupos de tipo E de las tierras bajas mayas representan lugares para rituales asociados con elementos astronómicos, especialmente los movimientos del sol que marcan equinoccios y solsticios (Aimers y Rice 2006; Aveni 2001; Aveni et al. 2003; Aveni y Hartung 1989). Esta interpretación implica que los Grupos de tipo E pudieron haber sido relacionados con el desarrollo de calendarios y concepciones del tiempo asociados con los movimientos del sol. Sin embargo, es importante notar que los Grupos de tipo E de Ceibal, del Chiapas y de La Venta son más tempranos que 


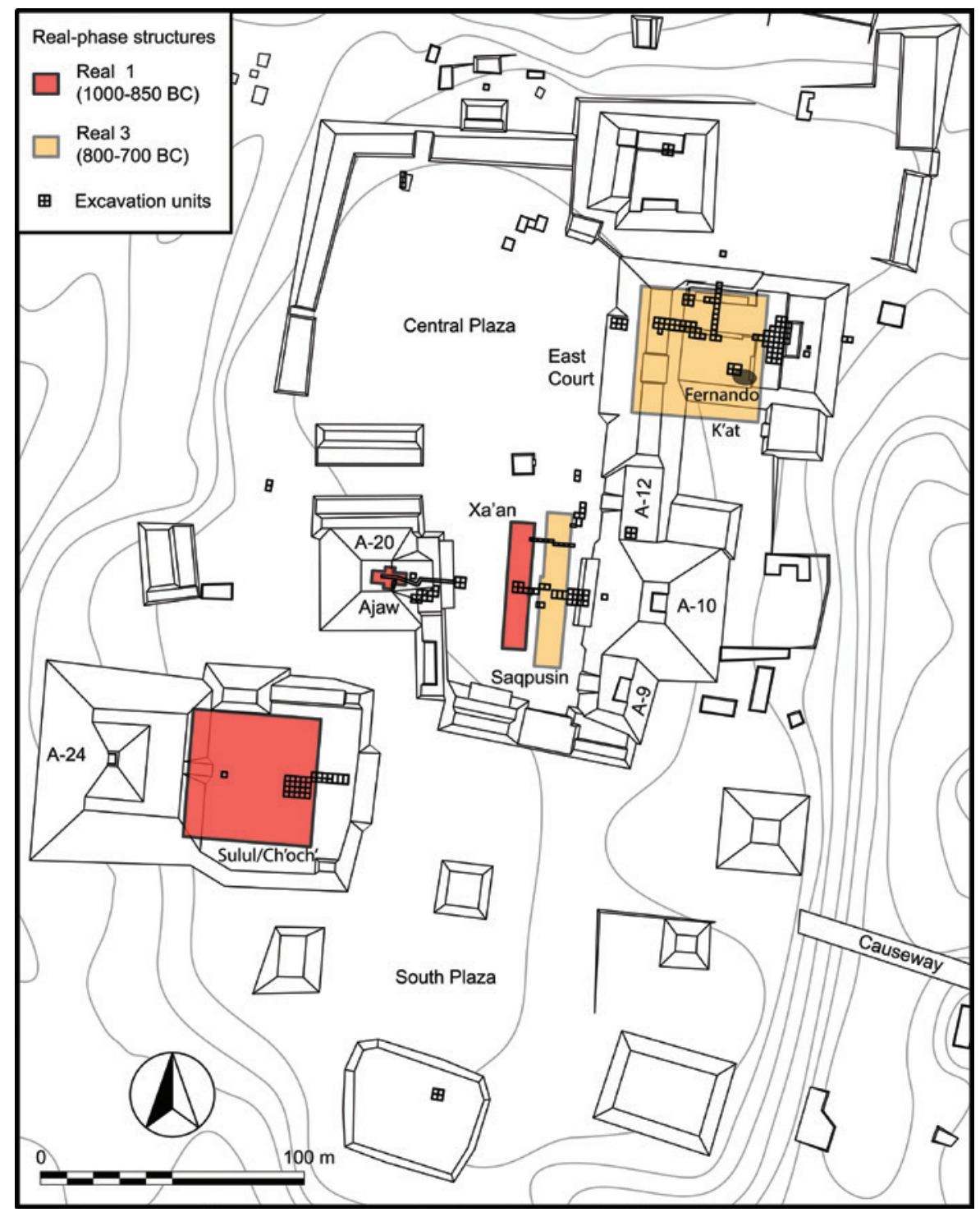

Fig. 3 - Mapa del Grupo A de Ceibal.

la mayoría de los conjuntos del mismo género de las tierras bajas mayas. El Grupo de tipo E confirmado más temprano en las tierras bajas mayas después de Ceibal es el de Cival, que se fecha hacia los años 800-700 a.C. (Estrada Belli 2006, 2011). El siguiente es el Mundo Perdido de Tikal, el cual se fecha más o menos de 700 a.C. (Laporte y Fialko 1995). 
Muchos Grupos de tipo E de Chiapas no se alinean particularmente con las direcciones del sol en los días de equinoccios o solsticios. En un estudio importante, Michael Blake (2013) indica que varios sitios chiapanecos con unas orientaciones generales norte-sur eran en realidad alineados con las direcciones de unos rasgos topográficos prominentes como montañas y cerros, habiendo sido incorporados también elementos de direcciones solares. Estas alineaciones con montañas son más claras en los sitios que tienen el plan de CFM o configuraciones similares en la costa Pacífico Sur, donde se encuentran volcanes altos en general hacia el norte. Por ejemplo, Izapa se orienta hacia el volcán Tacaná (Blake 2013), La Blanca hacia el volcán Tajumulco (Love y Guernsey 2011, p. 174), y Takalik Abaj hacia el volcán Siete Orejas (Schieber de Lavarreda, com. pers. 2015). Investigaciones recientes por Rosenswig et al. (2013) indican que Izapa también presenta un patrón CFM con un Grupo de tipo E, mientras que no queda claro si La Blanca y Takalik Abaj incluyeron Grupos de tipo E, eso debido a destrucciones modernas y a construcciones posteriores a los primeros edificios. Si Blake está en lo correcto, es posible que los Grupos de tipo E tempranos de Chiapas y de la costa Sur no hayan remitido exclusivamente a una concepción del tiempo universal vinculada con el sol, aun si pudo haber algunas relaciones con los movimientos cíclicos solares. En efecto, las principales referencias que estos conjuntos ceremoniales habrían tenido concernirían unos elementos terrenales del paisaje específico de cada área.

Los Grupos de tipo E de las tierras bajas mayas, incluyendo el de Ceibal, se aproximan más a la dirección este-oeste aunque muchos no corresponden exactamente con la posición del sol en los equinoccios y solsticios (Aimers y Rice 2006; Aveni y Hartung 1989; Aveni et al. 2003). Por otra parte, cabe subrayar que la mayoría de los sitios preclásicos en las tierras bajas mayas, con excepción de Ceibal, enfatizan el eje este-oeste en sus configuraciones espaciales, lo cual contrasta con la importancia del eje norte-sur en los sitios de Chiapas (Ashmore 1991; Ashmore y Sabloff 2002; Clark y Hansen 2001). Muchos de estos complejos mayas fueron construidos durante la parte tardía del Preclásico Medio o el Preclásico Tardío. Puede ser que estas configuraciones basadas en el eje este-oeste se deban, al menos parcialmente, a la ausencia de rasgos topográficos prominentes como montañas en esta región. Abundantes datos etnográficos confirman que los mayas modernos consideran la dirección este-oeste como el eje primario, correspondiendo al movimiento del sol, y los puntos norte y sur como orientaciones laterales de la línea principal (Bunzel 1952, p. 265; Gossen 1974, p. 31-32; Hanks 1990, p. 338; Vogt 1969, p. 603-604; Watanabe 1983; Wisdom 1940, p. 427). El énfasis en el eje este-oeste, presente aparentemente en la sociedad maya prehispánica y en grupos vecinos, tendría algo que ver con las condiciones topográficas de las tierras bajas mayas, habiendo sido seleccionado como eje direccional principal o, al menos, expresando más fuertemente el paso del sol. Esta ambigüedad en el 
carácter de los grupos de tipo E podría significar que los inicios de este género de conjunto arquitectónico no deben ser explicados solamente por su relación con unos calendarios o conceptos temporales vinculados con los movimientos del sol. Puede que su conexión con los ciclos del sol en realidad se incrementara a través de procesos históricos y tuviera que ver con factores idiosincráticos de situaciones locales.

Se puede proponer inferencias similares a partir del análisis de los escondites. Aquellos de Ceibal, del Chiapas y de La Venta incluyen rasgos cruciformes. Se ha sugerido que estas formas cruciformes estaban relacionadas con la direccionalidad cardinal (Ashmore 1991; McAnany 1995, p. 85; Schele y Freidel 1990). Nuestras investigaciones en Ceibal y comparaciones con los datos de otras áreas permiten proponer una cronología más detallada y precisa de ellos. Aunque los depósitos cruciformes pudieron haber aparecido durante la fase Real 1 (1000 a 850 a.C.), en su mayoría los primeros hallados en Ceibal y que se fechan de las fases Real 1 y 2 (1000 a 800 a.C.) no exhiben formas cruciformes (Aoyama et al. 2016; Inomata y Triadan 2016). Solamente un ejemplar de la fase Real 1 presenta una forma cruciforme, mientras que ocho con hachas de piedra verde de las fases Real 1 y 2 enseñan otras configuraciones: en ellas las hachas fueron puestas en posición horizontal formando una o varias líneas o una especie de flor (Figura 4, página siguiente). Estos arreglos tempranos son similares a los del Preclásico Temprano, es decir anteriores a 1000 a.C., que fueron encontrados en los sitios de El Manatí y La Merced situados cerca del centro olmeca temprano de San Lorenzo (Ortíz C. y Rodríguez 1999; Rodríguez y Ortíz C. 2000). Los escondites del Manatí y de La Merced se encuentran asociados con nacimientos naturales de agua, e implican una referencia a elementos terrenales. Aspectos de este simbolismo conectado con rasgos terrenales pudieron haberse mantenido en los escondites tempranos de Ceibal.

Escondites cruciformes se volvieron más comunes en la Mesoamérica del Sur después de 800 a.C. En Ceibal, cuatro depósitos en forma de cruz se fechan entre las fases Real 3 y Escoba 1 (800 a 600 a.C.) (Figura 5, página siguiente). Los escondites cruciformes identificados en Chiapas y en la costa del Golfo, incluyendo el Escondite 11 de San Isidro (Lowe 1981), la Ofrendas Masivas 1 y 2 y el Rasgo 3 de Chiapa de Corzo (Bachand y Lowe 2012) y la Ofrenda 10 de La Venta (Drucker et al. 1959), también se fechan después de 800 a.C. Además, el único escondite cruciforme con hachas de piedra verde en las tierras bajas mayas fuera de Ceibal, el Escondite 4 de Cival, se fecha después de 800 a.C. (Estrada Belli 2006, 2011).

Aunque las cosmologías mesoamericanas integran aspectos terrenales y celestiales de manera inseparable, es posible distinguir diferentes énfasis. En este sentido, los datos aquí referidos indican que el énfasis sobre la direccionalidad cardinal creció en la parte tardía de la tradición de los escondites con 
Takeshi INOMATA

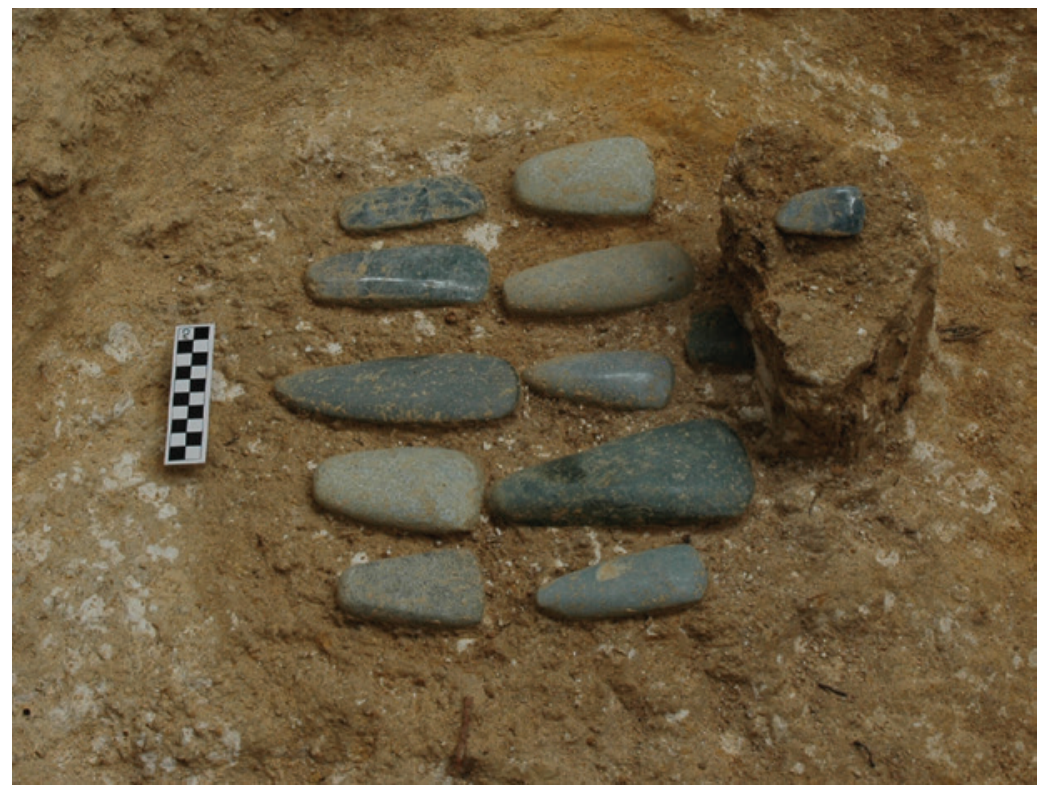

Fig. 4 - Escondite 118 que fue depositado alrededor de 1000 a.C.

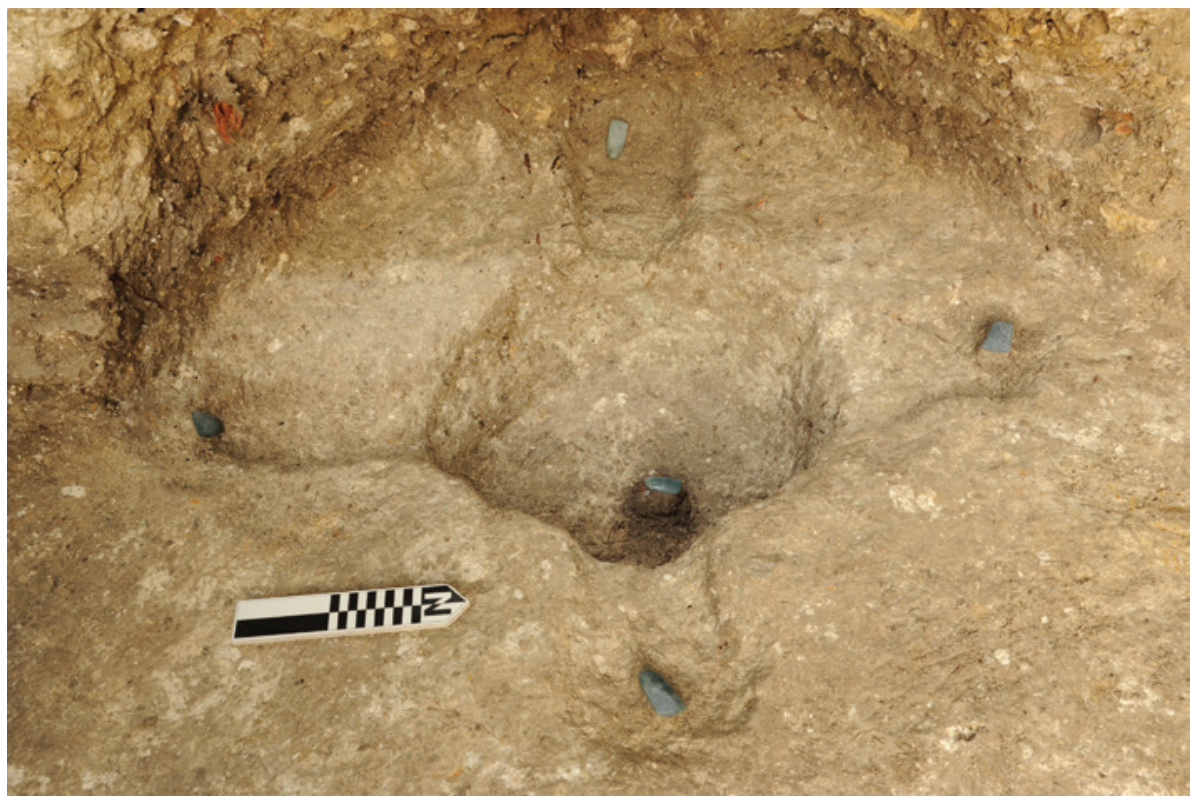

Fig. 5 - Escondite 160 que exhibe una configuración cruciforme y fue depositado probablemente entre 800 y 700 a.C. 
hachas de piedra verde. La cantidad de escondites cruciformes en las tierras bajas mayas está limitada; no obstante, el desarrollo de esta forma de depósito concuerda con la expansión de la configuración espacial de los sitios que privilegiaron la dirección este-oeste, un punto revelador de la importancia creciente del simbolismo del sol. A pesar de esta tendencia de mayor importancia de la referencia celestial, los grupos de tipo E y los escondites asociados no perdieron conexión con los elementos terrenales. Así pues, algunos escondites cruciformes de Ceibal (Escondites 7 y 171) y de Cival (Escondite 4) comportan por cierto cinco objetos principales $(4+1)$, pero son ollas para agua, $y$, aunque el agua puede remitir simbólicamente tanto al cielo como a la tierra, su presencia en depósitos con hachas indica más bien una conexión con los nacimientos de agua, es decir la tradición que se inició desde el Preclásico Temprano, por ejemplo en El Manatí o en La Merced.

El comienzo del desarrollo de los grupos de tipo E alrededor de 1000 a.C. corresponde aproximadamente con el momento en el que el cultivo del maíz se incrementó significativamente (Arnold 2009; Blake et al. 1992). En este contexto, es posible que el simbolismo de los grupos de tipo E haya sido relacionado con el cultivo de maíz (Aimers y Rice 2006; Doyle 2012; Stanton y Freidel 2003). Si esta hipótesis es correcta, los grupos de tipo E podrían haber integrado no solamente un ciclo de rituales públicos, sino también los ritmos de la vida cotidiana de la gente que cultivaba el maíz y otros alimentos. Ahora bien, cabe observar que, durante la fase temprana del Preclásico Medio, grupos que mantuvieron un modo de vida tradicional con fuerte dependencia en la caza y la recolección y con alta movilidad posiblemente coexistieron con los que adoptaron el cultivo del maíz y el sedentarismo (Inomata et al. 2015a, 2015b; Lohse 2010; Rosenswig 2010, 2011). En este contexto de heterogeneidad cultural marcada, los grupos de tipo E pueden haber remitido a distintos conceptos del tiempo, basados tanto en los ciclos del cultivo del maíz como en las temporalidades de la caza, la recolección y la pesca. La práctica de depositar hachas de piedra verde en los grupos de tipo E sugiere que el simbolismo del período anterior asociado con el sistema de subsistencia tradicional continuó durante el Preclásico Medio cuando surgieron nuevos conceptos relacionados con la agricultura del maíz.

\section{Cambios en los rituales durante el Preclásico Tardío}

Al final del Preclásico Medio, alrededor de 400 a.C., La Venta y varios sitios del Valle del Grijalva en Chiapas perdieron importancia. Con este cambio, la tradición de los grupos de tipo E en la costa del Golfo, en Chiapas y en la costa del Pacífico casi terminó. En la costa Pacífica, la ocupación continuó, pero Tzutzuculi, un sitio con un Grupo de tipo E y otros elementos del patrón 
de CFM, fue abandonado (McDonald 1983). En Izapa, aun si el Grupo de tipo E fue mantenido, el foco principal de actividad pasó a otros complejos (Lowe et al. 1982; Rosenswig et al. 2014). En realidad, los Grupo de tipo E nunca recuperaron su importancia social en la región del Istmo. En cambio, los mayas de las tierras bajas se volvieron los constructores más activos de los grupos de tipo E en Mesoamérica. Numerosos conjuntos de ese género fueron edificados allí durante el Preclásico Tardío, muchos de ellos enfatizando en aquel entonces el eje este-oeste en vez de la dirección norte-sur del período anterior. En Ceibal, los depósitos con hachas de piedra verde se volvieron poco comunes durante la fase Escoba de la parte tardía del Preclásico Medio (700-350 a.C.). Aun si la configuración general del sector que se discutió con anterioridad fue conservada, el patrón de su uso cambió significativamente a principios del Preclásico Tardío o fase Cantutse 1 (350-300 a.C.). Así pues, las plataformas grandes al noreste y al suroeste del Grupo de tipo E, que servían de bases para complejos residenciales en la parte tardía del Preclásico Medio, perdieron sus funciones domésticas. Por otra parte, muchos de los escondites que fueron depositados en la plaza del Grupo de tipo E durante la transición entre el Preclásico Medio y el Preclásico Tardío contuvieron en aquel momento, y por primera vez, restos de personajes sacrificados. Ahora bien, la relación de estos sacrificados con conceptos temporales es difícil de establecer.

Durante el Preclásico Tardío, el modo de vida con alta dependencia en la agricultura del maíz y en el sedentarismo ya se había plenamente difundido en las tierras bajas mayas. El simbolismo de los grupos de tipo E en esa época podría haberse relacionado con el ciclo del cultivo de maíz, pero los depósitos de restos de sacrificados en la plaza parecen reflejar más bien preocupaciones políticas, involucrando conflictos con comunidades vecinas.

\section{Posibles rituales calendáricos en el Preclásico Terminal}

Durante el Preclásico Terminal en Ceibal (100 a.C.-200 d.C.), se empezaron a depositar escondites de un nuevo tipo, los cuales pudieron haber reflejado conceptos temporales más específicamente vinculados con unos calendarios organizados sistemáticamente. En la parte este de la plaza del Grupo de tipo E, se hallaron numerosos escondites con vasijas cerámicas. Todas las vasijas son del tipo Sierra Rojo o de tipos parecidos con su característico acabado de engobe rojo ceroso, y no hay ningún tipo negro, crema o polícromo. Algunos escondites, sin embargo, contienen platos tetrápodes grandes, los cuales son diagnósticos de la etapa cerámica del Protoclásico. Las formas de los tetrápodes son altas y delgadas, y difieren de los característicos mamiformes (Figura 6, páginas siguientes). Otros escondites incluyeron platos con base plana y pared divergente y platos con base anular -ambas formas continuarán durante el Clásico 
Temprano. Se notan verdaderas secuencias estratigráficas entre algunos de los escondites. Por ejemplo, el Escondite CB103, que penetraba desde el Piso 5 o estaba sellado por él, cortó parte del Escondite CB104 que estaba sellado por el Piso 6 (Figura 7, página siguiente). El Escondite CB103 también estaba situado directamente encima del Escondite CB115. El análisis cerámico y las fechas de radiocarbono indican que los Escondites CB104 y 115, que contenían vasijas tetrápodes con soportes altos, se fechan de la fase Xate 2 (50-125 d.C.) mientras que los Escondites CB102 y 103, sin vasijas tetrápodes, pueden fecharse de la fase Xate 3 (125-200 d.C.). En varios depósitos, vasijas fueron puestas una encima de otra en varias capas, algunas incluyendo platos tetrápodes. Formaban a veces pares, al estar dispuestas labio contra labio, en la manera en que se multiplicarán los escondites del Clásico Temprano. En su mayoría las vasijas parecen haber sido enterradas completas, pero en algunos casos ciertas vasijas parecen haber sido quebradas intencionalmente antes de ser depositadas.

Varios escondites (CB102, 103, 104 y 119) contenían piedras de caliza trabajadas en forma de cilindros bajos. En el Escondite CB104, una fue colocada en el centro encima de un plato y otras tres en tres de las direcciones cardinales (Figura 7); no se encontró la cuarta pieza del lado oeste, pero esa pudo haber sido removida cuando se depositó el Escondite CB103.

En otros escondites, las piedras de este tipo no muestran patrones claros. Es difícil entender su función y significado, pero, por sus formas, parecen mini-altares. Muchos escondites, incluyendo los que no tenían vasijas cerámicas, contenían esferas de piedras del tamaño aproximado de una pelota de golf. Muchas son de piedra caliza y algunas parecen quemadas. También se localizó un pequeño número de cantos de río. Muchas esferas habían sido dispuestas dentro de vasijas, particularmente en las que formaban pares labio contra labio. Por ejemplo, cada par de vasijas del Escondite CB107 contenía siete esferas (Figura 8, páginas siguientes). En otros casos, se hallaron nueve o más. El plato superior del Escondite CB1 10 contaba 155 esferas (Figura 6). La función y el significado de estas piedritas nos escapan, pero una posible interpretación sería que se tratara de implementos para la adivinación o unos cálculos calendáricos.

Elementos comparables fueron reportados en Chiapas, en el altiplano maya, en la región del Chixoy en particular, y en la costa Pacífica, incluyendo el sitio de Takalik Abaj (Cheek 1977; Hicks y Rosaire 1960; Ichon y Arnauld 1985; Lowe y Agrinier 1960; Lowe 1962; Schieber de Lavarreda 2002; Schieber de Lavarreda y Orrego Corzo 2010; Schieber de Lavarreda y Pérez 2005; Sharer y Sedat 1987). Estos depósitos no son comunes en las tierras bajas mayas, aunque algunos ejemplos han sido reportados (Götting 2011; McAnany 2004; Źrałka et al. 2011). El patrón de distribución de los escondites que observamos sugiere que Ceibal reanudó durante este período las relaciones con Chiapas y con las tierras altas, relaciones que, en épocas anteriores, se habían expresado en ciertas configuraciones espaciales de los sitios y en los escondites con piedra verde. 
Takeshi INOMATA

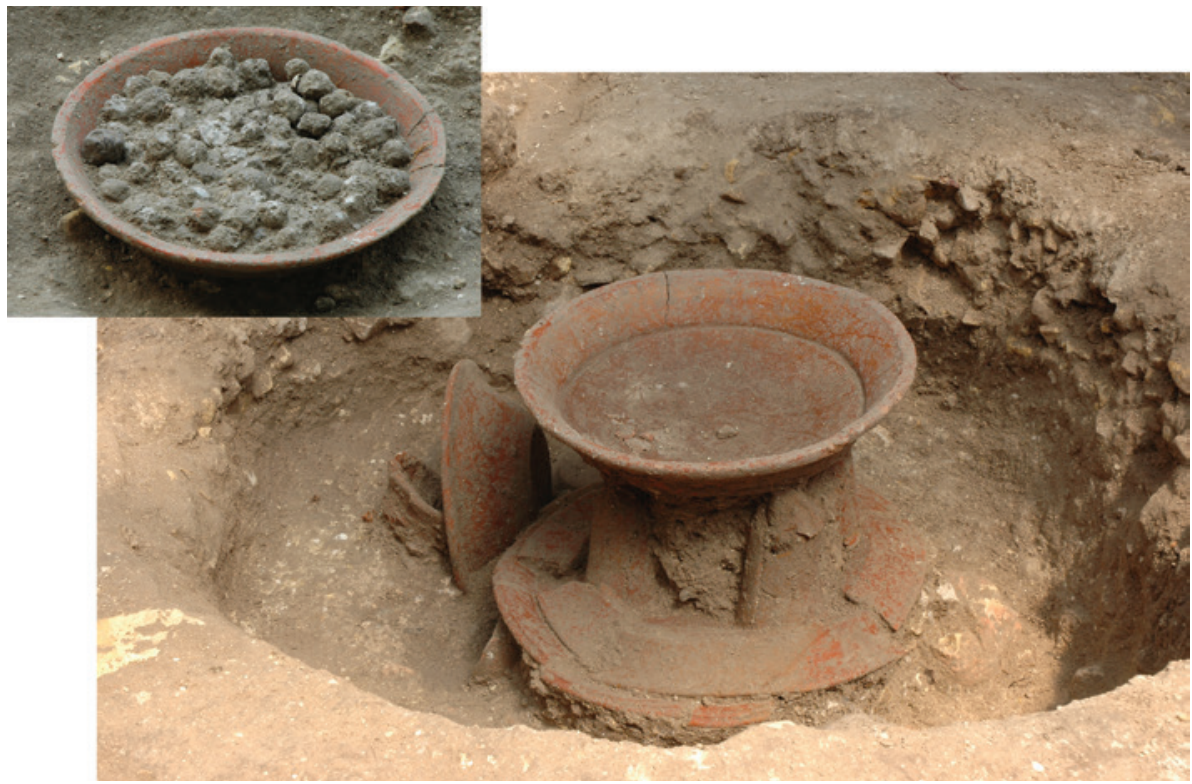

Fig. 6 - Escondite 110 del Preclásico Terminal que incluye vasijas tetrápodes altos. El plato superior contenía 155 esferas de piedra.

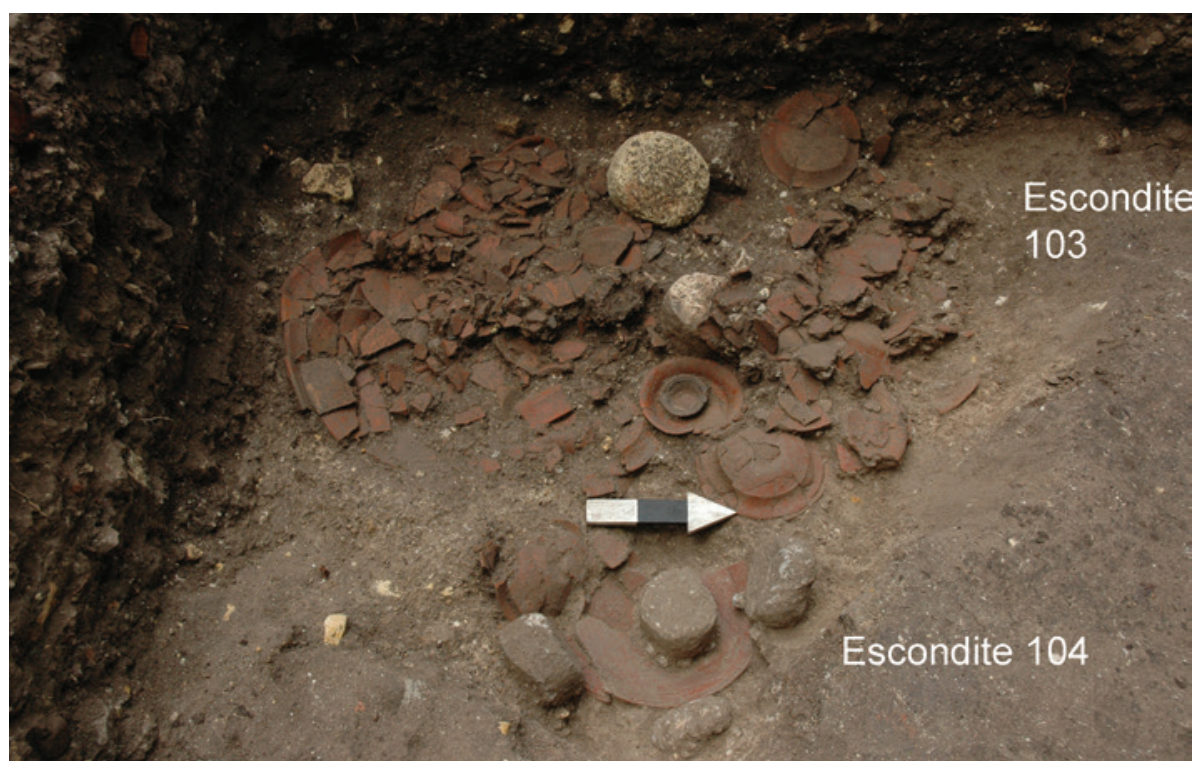

Fig. 7 - Escondites 103 y 104 del Preclásico Terminal. El Escondite 103 corta una parte del Escondite 104. 


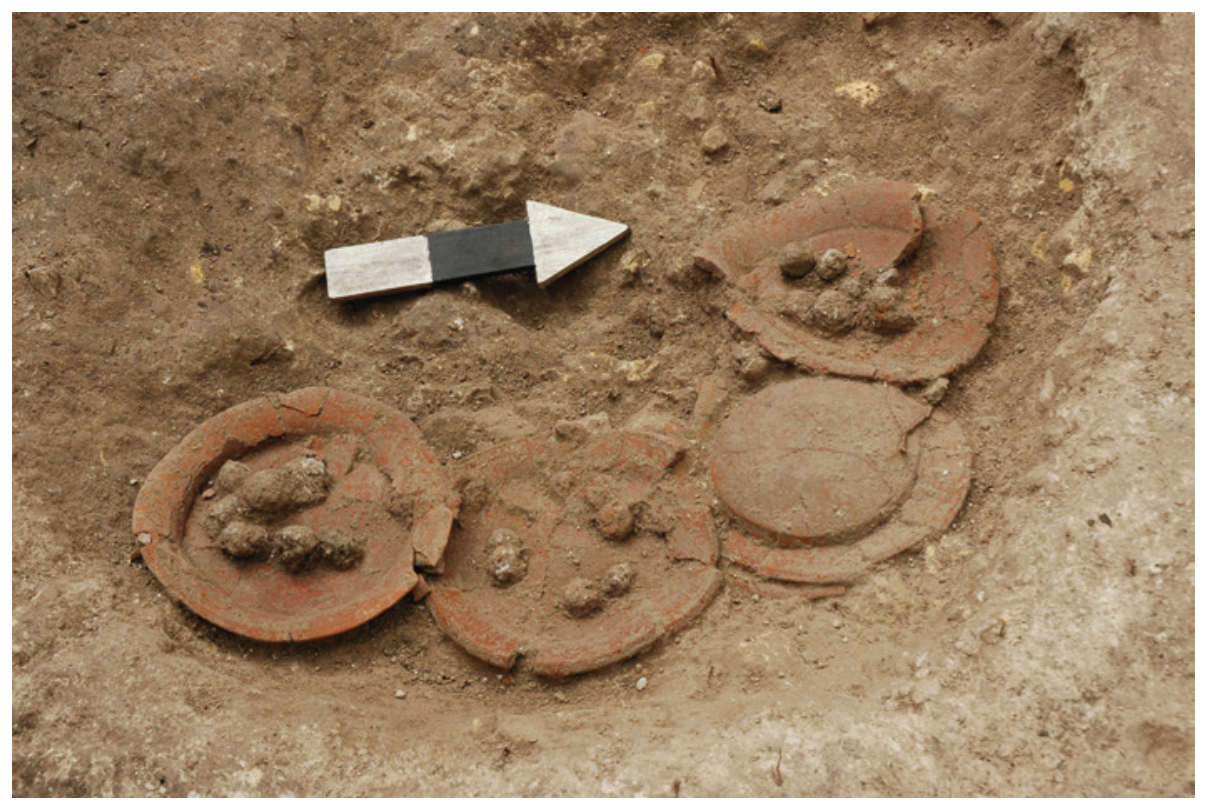

Fig. 8 - Escondite 107 del Preclásico Terminal. Cada pareja de platos que fueron colocados en una posición labio a labio contenía siete esferas de piedra.

Los depósitos del Preclásico Terminal de Ceibal son claramente secuenciales y posiblemente cíclicos. Es tentador pensar que estos escondites fueron dispuestos durante ceremonias calendáricas que se celebraban a intervalos regulares. Si fue el caso, estos depósitos implicarían que el Grupo de tipo E de Ceibal era entonces claramente conectado con unos conceptos temporales que compartían los miembros de la comunidad, participantes de estas ceremonias. Los ritos y depósitos realizados durante este período podrían haber presagiado las prácticas del mero Clásico en las cuales la conexión entre el Grupo de tipo E-los rituales celebrados en ese conjunto arquitectónico-y los ciclos calendáricos se expresó más explícitamente a través de los monumentos con inscripciones, los cuales conmemoran en particular los fines de los katun (20 años).

\section{Conclusión}

Los datos aquí recabados indican que las prácticas rituales en Ceibal y en otros sitios de la Mesoamérica del Sur estuvieron relacionadas con unos conceptos del tiempo así como con calendarios. Sin embargo, esas relaciones no fueron uni-direccionales. Así pues, no se puede aseverar que las concepciones temporales existentes en un momento determinaron las prácticas rituales y la 
edificación de estructuras arquitectónicas o de arreglos espaciales. Las prácticas sociales también afectaron las construcciones de conceptos simbólicos y temporales y, a su vez, estos conceptos abstractos condicionaron los procesos rituales y los de otros actos sociales. Las prácticas y experiencias físicas de la gente inevitablemente incorporan aspectos tanto repetitivos como secuenciales.

En el caso de los grupos de tipo E de Ceibal y de otros sitios, estos complejos arquitectónicos pudieron incorporar diversos tipos de conceptos y prácticas, inclusive referencias a rasgos topográficos y a puntos temporales marcados por los movimientos del sol y de otras entidades celestiales. El simbolismo de los elementos terrenales y celestiales pudieron coexistir a lo largo de la historia de los grupos de tipo E, aunque es posible discernir cambios en el énfasis puesto en tal o cual aspecto. En la etapa inicial del desarrollo de los grupos de tipo E, las referencias a rasgos naturales del paisaje local de cada región parecen haber sido importantes. Durante las partes media y tardía del período Preclásico Medio, especialmente con la difusión de los grupos de tipo E en las tierras bajas mayas, la conexión de estos conjuntos arquitectónico con los movimientos del sol pasó al primer plano. Fuera lo que fuera de estos cambios, queda claro que distintos conceptos de tiempo y espacio fueron incorporados en las formas construidas y en los rituales asociados.

Las diferentes referencias y representaciones referidas pueden haberse basado en las experiencias cotidianas de la gente y de sus diferentes ritmos de vida, incluyendo los correlativos al cultivo de maíz como aquellos vinculados con las actividades de caza, recolección y pesca. Si quienes critican a Bloch acerca de la distinción que hace entre tiempo ritual y tiempo mundano están en lo correcto, queda por precisar cómo la temporalidad de los rituales públicos y aquellas de los diferentes modos de vida se integraron en la concepción del tiempo de cada población. Los rituales públicos y los proyectos de construcción monumental probablemente ofrecieron oportunidades para compartir un mismo sentido de la temporalidad entre diversos miembros de una comunidad dada, los cuales seguían diferentes ritmos de vida en otros contextos. Las experiencias y los entendimientos de la gente que participó en la edificación de grupos de tipo E y en los eventos públicos que ahí fueron celebrados, posiblemente involucraron aspectos tanto cíclicos como secuenciales. Las dimensiones cíclicas pueden haber sido expresadas por ciclos de rituales y depósitos asociados, mientras que las secuenciales fueron materializadas por las acumulaciones continuas de los pisos de la plaza y las construcciones de edificios. Si estas prácticas y experiencias afectaron los conceptos temporales, las concepciones que resultaron no pueden ser vistas exclusivamente como cíclicas o lineales.*

* Manuscrit reçu en décembre 2015, accepté pour publication en octobre 2016.

Este artículo forma parte del número especial Compases y texturas del tiempo entre los mayas: lo dicho, lo escrito, lo vivido. 
Agradecimientos - Las investigaciones en Ceibal fueron realizadas con la autorización del Instituto de Antropología e Historia (IDAEH) de Guatemala. Apoyos financieros fueron aportados por National Geographic Society, National Endowment for the Humanities (RZ-51209-10) y Alphawood Foundation en el caso de Takeshi Inomata y Daniela Triadan. Por su parte, el ministerio de Educación, Cultura, Deportes, Ciencias y Tecnología de Japón KAKENHI (21101003 and 21101002) y la Sociedad japonesa para la promoción de la Ciencia KAKENHI (21402008, 26101002, and 26101003) apoyaron a Kazuo Aoyama. Parte de este manuscrito fue redactada durante mi estancia en Dumbarton Oaks apoyada por una beca. Agradezco a Víctor Castillo la revisión del español.

\section{Referencias citadas}

Agrinier Pierre

1975 Mounds 9 and 10 at Mirador, Chiapas, Mexico, Papers of the New World Archaeological Foundation, n 39, Brigham Young University, Provo (UT).

2000 Mound 27 and the Middle Preclassic Period at Mirador, Chiapas, Mexico, Papers of the New World Archaeological Foundation, $n^{\circ}$ 58, Brigham Young University, Provo (UT).

Aimers James J. y Prudence M. Rice

2006 “Astronomy, Ritual, and the Interpretation of Maya 'E-Group' Architectural Assemblages", Ancient Mesoamerica, 17 (1), p. 79-96.

Aoyama Kazuo, Takeshi Inomata, Flory Pinzón y Juan Manuel Palomo

2016 "The Development of Maya Civilization and Public Rituals of the Preclassic Maya: Polished Greenstone Celt Caches from Ceibal, Guatemala", Antiquity.

ARnold Philip J., III

2009 "Settlement and Subsistence among the Early Formative Gulf Olmec", Journal of Anthropological Archaeology, 28, p. 397-411.

AsHMORE Wendy

1991 "Site-Planning Principles and Concepts of Directionality among the Ancient Maya", Latin American Antiquity, 2 (3), p. 199-226.

Ashmore Wendy y Jeremy A. SABLofF

2002 "Spatial Orders in Maya Civic Plans", Latin American Antiquity, 13 (2), p. 201-215.

Aveni Anthony F.

2001 Skywatchers, University of Texas Press, Austin.

Aveni Anthony y Horst Hartung

1989 "Uaxactun, Guatemala, Group E and Similar Assemblages: Archaeoastronomical Reconsideration", in Anthony Aveni (ed.), World Archaeoastronomy, Cambridge University Press, Cambridge, p. 441-461.

Aveni Anthony F., Anne S. Dowd y Benjamin Vining

2003 "Maya Calendar Reform? Evidence from Orientations of Specialized Architectural Assemblages", Latin American Antiquity, 14 (2), p. 159-178. 
BACHAND Bruce R. y Lynneth S. Lowe

2012 "Chiapa de Corzo's Mound 11 Tomb and the Middle Formative Olmec", in Lynneth S Lowe y Mary E. Pye (eds), Arqueología reciente de Chiapas: contribuciones del Encuentro celebrado en el 60 aniversario de la Fundación arqueológica Nuevo Mundo, Papers of the New World Archaeological Foundation, $\mathrm{n}^{\circ} 72$, Brigham Young University, Provo (UT), p. 45-68.

BLAKE Michael

2013 "Solar Orientations and Formative Period Site Layouts in SE Mesoamerica: Sunrise and Sunset Alignments during the Equinoxes and Solstices", paper presented at the 78th annual meeting of the Society for American Archaeology, Honolulu.

Blake Michael, John E. Clark, Barbara Voorhies, Michael W. Love y Brian S. Chisholm

1992 "Prehistoric Subsistence in the Soconusco Region", Current Anthropology, 33, p. 83-94.

BLOCH Maurice

1977 "The Past and the Present in the Present", Man, N.S. 12 (2), p. 278-292.

BRADLEY Richard

1991 "Ritual, Time and History", World Archaeology, 23 (2), p. 209-219.

Bunzel Ruth L.

1952 Chichicastenango. A Guatemalan Village, University of Washington Press, Seattle. CHeEk Charles

1977 "Excavations at the Palangana and the Acropolis, Kaminaljuyu", in William T. Sanders y Joseph W. Michels (eds), Teotihuacan and Kaminaljuyu. A Study in Prehistoric Culture Contact, Pennsylvania State University Press, University Park, p. 1-204.

Clark John E. y Richard D. HANSEN

2001 "Architecture of Early Kingship: Comparative Perspectives on the Origins of the Maya Royal Court", in Takeshi Inomata and Stephen D. Houston (eds), Royal Courts of the Ancient Maya, Westview Press (Data and Case Studies, 2), Boulder, p. 1-45.

Doyle James

2012 “Regroup on 'E-Groups': Monumentality and Early Centers in the Middle Preclassic Maya Lowlands”, Latin American Antiquity, 23 (4), p. 355-379.

Drucker Philip, Robert F. Heizer y Robert H. SQuier

1959 Excavations at La Venta, Tabasco, Bureau of American Ethnology Bulletin, Smithsonian Institution, Washington, D.C, vol. 170.

Estrada Belli Francisco

2006 "Lightning Sky, Rain, and the Maize God. The Ideology of Preclassic Maya Rulers at Cival, Peten, Guatemala", Ancient Mesoamerica, 17 (1), p. 57-78.

2011 The First Maya Civilization: Ritual and Power before the Classic Period, Routledge, London.

Gell Alfred

1992 The Anthropology of Time: Cultural Constructions of Temporal Maps and Images, Berg, Oxford. 
Entre rutinas cotidianas y eventos extraordinarios

Gossen Gary H.

1974 Chamulas in the World of the Sun: Time and Space in a Maya Oral Tradition, Harvard University Press, Cambridge (MA).

GötTING Eva

2011 "Excavaciones en el Edificio H-XVI (Operación 12)”, in Milan Kováč y Ernesto Arredondo Leiva (eds), Proyecto Arqueológico SAHI-Uaxactun Informe $n^{\circ}$ 2. Temporada de campo 2010, Instituto de Antropología e Historia de Guatemala (Guatemala), p. 479-504.

Graham John A.

1990 Excavations at Seibal, Department of Peten, Guatemala: Monumental Sculpture and Hieroglyphic Inscriptions, Memoirs of the Peabody Museum of Archaeology and Ethnology, Gordon R. Willey (series editor, vol. 14, n 1), Harvard University, Cambridge (MA).

HANKS William F.

1990 Referential Practice: Language and Lived Space among the Maya, University of Chicago Press, Chicago.

Hicks Frederick y Charles E. RosAIRE

1960 Mound 13, Chiapa De Corzo, Chiapas, Mexico, Papers of the New World Archaeological Foundation, n 10 , Brigham Young University, Provo (UT).

Howe Leopold E.A.

1981 "The Social Determination of Knowledge: Maurice Bloch and Balinese Time", Man, N.S., 16 (2), p. 220-234.

ICHON Alain y Marie-Charlotte ARNAULD

1985 Le Protoclassique à La Lagunita, El Quiché, Guatemala, Centre National de la Recherche Scientifique, Institut d'Ethnologie, Paris.

INOMATA Takeshi

2014a "War, Violence, and Society in the Maya Lowlands", in Andrew Scherer y John Verano (eds), Embattled Bodies, Embattled Places: War in Pre-Columbian America, Dumbarton Oaks Research Library and Collection, Washington (DC), p. 25-56.

2014b "Plaza Builders of the Preclassic Maya Lowlands: The Construction of a Public Space and a Community at Ceibal, Guatemala", in Kenishiro Tsukamoto y Takeshi Inomata (eds), Mesoamerican Plazas. Practices, Meanings, and Memories, University of Arizona Press, Tucson, p. 19-34.

InOMATA Takeshi y Daniela TRIADAN

2016 "Middle Preclassic Caches from Ceibal, Guatemala", Maya Archaeology.

Inomata Takeshi, Jessica MacLellan y Melissa Burham

2015a "The Construction of Public and Domestic Spheres in the Preclassic Maya Lowlands", American Anthropologist, 117 (3), p. 519-534.

Inomata Takeshi, Jessica MacLellan, Daniela Triadan, Jessica Munson, Melissa Burham, Kazuo Aoyama, Hiroo Nasu, Flory Pinzón y Hitoshi Yonenobu

2015b "Development of Sedentary Communities in the Maya Lowlands: Coexisting Mobile Groups and Public Ceremonies at Ceibal, Guatemala", Proceedings of the National Academy of Sciences of the United States of America, 112 (14), p. $4268-4273$. 
InOmata Takeshi, Daniela Triadan y Otto Rodrigo Román

2010 "La transformación y continuidad de ritos durante el período Preclásico en Ceibal, Guatemala", in Andrés Ciudad Ruiz, María Josefa Iglesias Ponce de León y Miguel Angel Sorroche (eds), El Ritual en el mundo maya. De lo privado a lo público, Sociedad española de estudios mayas, Madrid, p. 29-48.

Inomata Takeshi, Daniela Triadan, Kazuo Aoyama, Victor Castillo y Hitoshi Yonenobu

2013 "Early Ceremonial Constructions at Ceibal, Guatemala, and the Origins of Lowland Maya Civilization", Science, 340 (6131), p. 467-471.

LAPORTE Juan Pedro y Vilma Fialko

1995 "Reencuentro con Mundo Perdido, Tikal, Guatemala", Ancient Mesoamerica, $6(1)$, p. 41-94.

LoHse Jon C.

2010 "Archaic Origins of the Lowland Maya", Latin American Antiquity, 21, (3), p. 312-352.

LOVE Michael y Julia GuernSEY

2011 "La Blanca and the Soconusco Middle Formative", in Richard G. Lesure (ed), Early Mesoamerican Social Transformations: Archaic and Formative Lifeways in the Soconusco Region, University of California Press, Berkeley, p. 170-188.

Lowe Gareth W.

1962 Mound 5 and Minor Excavations, Chiapa De Corzo, Chiapas, Mexico, Papers of the New World Archaeological Foundation, n 12, Brigham Young University, Provo (UT).

1977 "The Mixe-Zoque as Competing Neighbors of the Early Lowland Maya", in Richard E. W. Adams (ed.), The Origins of Maya Civilization, University of New Mexico Press, Albuquerque, p. 197-248.

1981 "Olmec Horizon Defined in Mound 20, San Isidro, Chiapas", in Michael D. Coe y David Grove (orgs), Elizabeth P. Benson (ed.), The Olmec and their Neighbors, Dumbarton Oaks Research Library and Collection, Washington (DC), p. 231-256.

Lowe Gareth W. y Pierre Agrinier

1960 Mound 1, Chiapa De Corzo, Chiapas, Mexico, Papers of the New World Archaeological Foundation, $\mathrm{n}^{\circ}$ 8, Brigham Young University, Provo (UT).

Lowe Gareth W., Thomas A. LeE y Eduardo E. MARTíneZ

1982 Izapa. An Introduction to the Ruins and Monuments, Papers of the New World Archaeological Foundation, no 31, Brigham Young University, Provo (UT).

Mason J. Alden

1960 Mound 12, Chiapa De Corzo, Chiapas, Mexico. Papers of the New World Archaeological Foundation, $n^{\circ}$ 9, Brigham Young University, Provo (UT).

McAnany Patricia A.

1995 Living with the Ancestors. Kinship and Kingship in Ancient Maya Society, University of Texas Press, Austin.

McAnany Patricia A. (ed.)

2004 K'axob: Ritual, Work and Family in an Ancient Maya Village, Monumenta Archaeologica, University of California at Los Angeles, Cotsen Institute of Archaeology, Los Angeles, vol. 22. 
Entre rutinas cotidianas y eventos extraordinarios

McDonald Andrew J.

1983 Tzutzuculi: A Middle-Preclassic Site on the Pacific Coast of Chiapas, Mexico, Papers of the New World Archaeological Foundation, n 47, Brigham Young University, Provo (UT).

McDonald Andrew J.

1999 Middle Formative Pyramidal Platform Complexes in Southern Chiapas, Mexico: Structure and Meaning; tesis doctoral, University of Texas, Austin.

Miller Donald E.

2014 Excavations at La Libertad, a Middle Formative Ceremonial Center in Chiapas, Mexico, Papers of the New World Archaeological Foundation, $n^{\circ}$ 64, Brigham Young University, Provo (UT).

MunN Nancy D.

1992 "The Cultural Anthropology of Time: A Critical Essay", Annual Review of Anthropology, 21, p. 93-123.

OrTíz C. Ponciano y María del Carmen Rodríguez

1999 "Olmec Ritual Behavior at El Manatí: A Sacred Space", in David C. Grove y Rosemary A. Joyce (eds), Social Patterns in Pre-Classic Meosamerica, Dumbarton Oaks Research Library and Collection, Washington (DC), p. 225-254.

Rodríguez María del Carmen y Ponciano OrTíz

2000 "A Massive Offering of Axes at La Merced, Hidalgotitlán, Veracruz, Mexico", in John E. Clark y Mary E. Pye (eds), Olmec Art and Archaeology in Mesoamerica, National Gallery of Art, Washington (DC), p. 155-167.

Rosenswig Robert M.

2010 The Beginnings of Mesoamerican Civilization. Inter-Regional Interaction and the Olmec, Cambridge University Press, Cambridge.

2011 "An Early Mesoamerican Archipelago of Complexity", in Richard G. Lesure (ed.), Early Mesoamerican Social Transformations: Archaic and Formative Lifeways in the Soconusco Region, University of California Press, Berkeley, p. 242-271.

Rosenswig Robert M., Ricardo López-Torrijos, Caroline E. Antonelli y Rebecca R. MENDELSOHN

2013 "LiDAR Mapping and Surface Survey of the Izapa State on the Tropical Piedmont of Chiapas, Mexico", Journal of Archaeological Science, 40 (3), p. 1493-1507.

Rosenswig Robert M., Ricardo LóPez-Torrijos y Caroline E. ANTONELli

2014 "LiDAR Data and the Izapa Polity: New Results and Methodological Issues from Tropical Mesoamerica", Archaeological and Anthropological Sciences [on-line], http://link.springer.com/article/10.1007/s12520-014-0210-7\#, consultado el 30 de septiembre de 2015.

SABLoff Jeremy A.

1975 Excavations at Seibal, Department of Peten, Guatemala: Ceramics, Memoirs of the Peabody Museum of Archaeology and Ethnology, Gordon R. Willey (series editor, vol. 13, $\mathrm{n}^{\circ}$ 2), Harvard University, Cambridge (MA).

Schele Linda y David A. Freidel

1990 A Forest of Kings. The Untold Story of the Ancient Maya, Morrow, New York. 
Schieber de LavarReda Christa

2002 "La Ofrenda de Abaj Takalik", in Juan Pedro Laporte, Héctor L. Escobedo y Barbara Arroyo (eds), XV Simposio de Investigaciones Arqueológicas en Guatemala, 2001, Museo Nacional de Arqueología y Etnología, Guatemala, p. 459-473.

Schieber de Lavarreda Christa y Miguel Orrego Corzo

2010 "Preclassic Olmec and Maya Monuments and Architecture at Takalik Abaj", in Julia Guernsey, John E. Clark y Barbara Arroyo (eds), The Place of Stone Monuments: Context, Use, and Meaning in Mesoamerica's Preclassic Tradition, Dumbarton Oaks Research Library and Collection, Washington (DC), p. 177-205.

Schieber de Lavarreda Christa y Geremías Claudio Pérez

2005 "La Ofrenda del incensario 'La Niña”, in Juan Pedro Laporte, Bárbara Arroyo y Héctor E. Mejía (eds), XVIII Simposio de Investigaciones Arqueológicas en Guatemala, 2004, Museo Nacional de Arqueología y Etnología, Guatemala, p. 751-762.

SHARER Robert J. y David W. SEDAT

1987 Archaeological Investigations in the Northern Maya Highlands, Guatemala: Interaction and the Development of Maya Civilization, University Museum Monograph, University Museum, University of Pennsylvania, Philadelphia, vol. 59.

SMith A. Ledyard

1982 Excavations at Seibal, Department of Peten, Guatemala: Major Architecture and Caches, Memoirs of the Peabody Museum of Archaeology and Ethnology, Gordon R. Willey (series editor, vol. 15, n 1), Harvard University, Cambridge (MA).

Stanton Travis W. y David A. Freidel

2003 "Ideological Lock-in and the Dynamics of Formative Religions in Mesoamerica", Mayab, 16, p. 5-14.

TOURTELLOT Gair III

1988 Excavations at Seibal, Department of Peten, Guatemala: Peripheral Survey and Excavation, Settlement and Community Patterns, Memoirs of the Peabody Museum of Archaeology and Ethnology, Gordon R. Willey (series editor, vol. 16), Harvard University, Cambridge (MA).

TREAT Raymond

1986 Early and Middle Formative Sub-Mound Refuse Deposits at Vistahermosa, Chiapas. Notes of the New World Archaeological Foundation, $\mathrm{n}^{\circ} 2$, Brigham Young University, Provo (UT).

Vogt Evon Z.

1969 Zinacantán. A Maya Community in the Highlands of Chiapas, Belknap Press of Harvard University Press, Cambridge.

Watanabe John M.

1983 "In the World of the Sun. A Cognitive Model of Mayan Cosmology", Man, N.S., 18 (4), p. 710-728. 
Entre rutinas cotidianas y eventos extraordinarios

WILLEY Gordon R.

1990 Excavations at Seibal, Department of Peten, Guatemala. General Summary and Conclusions, Memoirs of the Peabody Museum of Archaeology and Ethnology, no 4, Goré \& ${ }^{2} \&$ "' don R. Willey (series editor, vol. 17), Harvard University, Cambridge (MA).

WisDom Charles

1940 The Chorti Indians of Guatemala, University of Chicago Press, Chicago.

Źraeka Jarosław, Wiesław Koszkul, Simon Martin y Bernard Hermes

2011 "In the Path of the Maize God. A Royal Tomb at Nakum, Petén, Guatemala", Antiquity, 85, p. 890-908. 
